FACTA UNIVERSITATIS (NIŠ)

Ser. Math. Inform. Vol. 36, No 3 (2021), 489 - 499

https://doi.org/10.22190/FUMI200318036A

Original Scientific Paper

\title{
REGULAR FRACTIONAL DIRAC TYPE SYSTEMS
}

\author{
Bilender P. Allahverdiev ${ }^{1}$ and Hüseyin Tuna ${ }^{2}$ \\ ${ }^{1}$ Faculty of Science and Letter, Department of Mathematics, \\ Süleyman Demirel University, 32260 Isparta, Turkey \\ 2 Faculty of Science and Letter, Department of Mathematics \\ Mehmet Akif Ersoy University, 15030 Burdur, Turkey
}

\begin{abstract}
In this paper, we study one dimensional fractional Dirac type systems which include the right-sided Caputo and the left-sided Riemann-Liouvile fractional derivatives of the same order $\alpha, \alpha \in(0,1)$. We investigate the properties of the eigenvalues and the eigenfunctions of this system.

Keywords: Fractional Dirac system, Riemann-Liouville and Caputo derivatives
\end{abstract}

\section{Introduction}

It is well known that classical calculus is based on integer order differentiation and integration. Fractional calculus generalizes integrals and derivatives to noninteger orders. The subject has a long history. Since 1695, many mathematicians, among them Liouville, Riemann, Leibniz, Grunwald, Letnikov Riesz and Caputo, have studied this subject. Fractional calculus has important applications to many real-world phenomena studies in engineering, chemistry, mechanics, physics, finance, etc. There is an extensive literature on this subject, see for example $[9,10,16,17$, $19,20,22,23,24]$ and references therein.

Recently, the study of boundary value problems for fractional Sturm-Liouville equations recently has attracted a great deal of attention from many researchers. In

\footnotetext{
Received March 18, 2020. accepted April 06, 2021.

Communicated by Hari Mohan Srivastava

Corresponding Author: Bilender P. Allahverdiev, Faculty of Science and Letter, Department of Mathematics, Süleyman Demirel University, 32260 Isparta, Turkey | E-mail: bilenderpasaoglu@sdu.edu.tr

2010 Mathematics Subject Classification. Primary 26A33, 34A08, ; Secondary 34L40, 34B05, $47 \mathrm{~B} 25$

(C) 2021 by University of Niš, Serbia | Creative Commons License: CC BY-NC-ND
} 
[4], the authors investigated some basic spectral properties of the fractional SturmLiouville problem with Generalized Dirichlet conditions. They proved that this problem has an infinite sequence of real eigenvalues and the corresponding eigenfunctions form a complete orthonormal system in the Hilbert space $L_{2}[a, b]$. In [11], the authors studied the properties of the eigenfunctions and the eigenvalues of the regular Generalized Fractional Sturm-Liouville Problem. In [6], the authors studied the fractional Sturm-Liouville problem associated with the Weber fractional derivative of order $\alpha$. In [15], the authors proved existence of strong solutions for the space-time fractional diffusion equations. Using the method of separating variables, they solved several types of fractional diffusion equations. Klimek et al. [13] studied to the regular fractional Sturm-Liouville eigenvalue problem. By applying the methods of fractional variational analysis, they proved the existence of a countable set of orthogonal solutions and corresponding eigenvalues. Klimek and Argawal [12] defined some fractional Sturm-Liouville operators and introduced two classes of fractional Sturm-Liouville problems namely regular and singular fractional Sturm-Liouville problems. They investigated the eigenvalue and eigenfunction properties of this classes. Baş [2] gave the theory of spectral properties for eigenvalues and eigenfunctions of Bessel type of fractional singular Sturm-Liouville problem. Baş and Metin [3] studied a fractional singular Sturm-Liouville operator having Coulomb potential of type. Klimek and Blasik [14] studied a regular fractional Sturm-Liouville problem with left and right Liouville-Caputo derivatives of order in the range $(1 / 2,1)$. They proved that it has an infinite countable set of positive eigenvalues and its continuous eigenvectors form a basis in the space of square-integrable functions. Rivero et al. [21] studied some of the basic properties of the fractional version of the Sturm-Liouville problem. Zayernouri and Karniadakis [27] studied new classes of the regular and singular fractional Sturm-Liouville Problems and obtained some explicit forms of the eigenfunctions.

While the theory of fractional Sturm-Liouville equations is well developed, the literature involving fractional Dirac system is scarce. In [7], Ferreira and Vieira derived fundamental solutions for the fractional Dirac operator which factorizes the fractional Laplace operator. In [8], the authors obtained eigenfunctions and fundamental solutions for the three parameter fractional Laplace operator defined via fractional Liouville-Caputo derivatives. They also obtained a family of fundamental solutions of the corresponding fractional Dirac operator. In [5], the author proved Lieb-Thirring type bounds for fractional Schrödinger operators and Dirac operators with complex-valued potentials. In [1], the authors studied a regular $q$-fractional Dirac type system. In the present paper, we consider the fractional Dirac type system defined by

$$
\left(\begin{array}{cc}
0 & { }^{C} D_{b^{-}}^{\alpha} \\
D_{a^{+}}^{\alpha} & 0
\end{array}\right)\left(\begin{array}{l}
y_{1} \\
y_{2}
\end{array}\right)+\left(\begin{array}{cc}
p(x) & 0 \\
0 & r(x)
\end{array}\right)\left(\begin{array}{l}
y_{1} \\
y_{2}
\end{array}\right)=\lambda\left(\begin{array}{l}
\omega_{1} y_{1} \\
\omega_{2} y_{2}
\end{array}\right)
$$

where $p, r, \omega_{1}$ and $\omega_{2}$ are real-valued continuous functions defined on $[a, b]$ and $\omega_{i}(x)>$ $0, \forall x \in[a, b],(i=1,2), \lambda$ is a complex spectral parameter. If we take $\alpha \rightarrow 1$ in this system, then we get the one dimensional Dirac type system. This system is one of the basic models of one-dimensional quantum mechanics. For example, a 
relativistic electron in the electrostatic field $\Omega(x)$ is described by the system

$$
\left(\begin{array}{cc}
0 & -\frac{d}{d x} \\
\frac{d}{d x} & 0
\end{array}\right) f(x)+\left(\begin{array}{cc}
\Omega(x)-\frac{m c}{h} & k x^{-1} \\
k x^{-1} & \Omega(x)+\frac{m c}{h}
\end{array}\right) f(x)=\frac{\lambda}{h c} f(x)
$$

where $c>0$ is the velocity of light, $k \in \mathbb{Z} \backslash\{0\}, \Omega(x)$ is a spherically symmetric potential, $m>0$ is the mass of the particle ([26]). Basic properties of the one dimensional Dirac systems have been considered in [18], [26], [25] and the references therein.

\section{Preliminaries}

In this section, we provide some basic definitions and properties of the fractional calculus theory. These concepts and properties can be found in [20],[16],[22],[10], and references therein.

Definition 2.1. (see [20]) Let $0<\alpha \leq 1$ and $f \in L_{1}(a, b)$. The right-sided and left-sided Riemann-Liouville integrals of order $\alpha$ are given by the formulas, respectively

$$
\begin{aligned}
& \left(I_{b^{-}}^{\alpha} f\right)(x)=\frac{1}{\Gamma(\alpha)} \int_{x}^{b} f(s)(s-x)^{\alpha-1} d s, \quad x<b, \\
& \left(I_{a^{+}}^{\alpha} f\right)(x)=\frac{1}{\Gamma(\alpha)} \int_{a}^{x} f(s)(x-s)^{\alpha-1} d s, \quad x>a,
\end{aligned}
$$

where $\Gamma$ denotes the gamma function.

Definition 2.2. (see [20]) Let $0<\alpha \leq 1$ and $f \in L_{1}(a, b)$. The right-sided and respectively left-sided Riemann-Liouville derivatives of order $\alpha$ are defined, respectively, as follows

$$
\begin{aligned}
& \left(D_{b^{-}}^{\alpha} f\right)(x)=-D\left(I_{b^{-}}^{1-\alpha} f\right)(x), x<b, \\
& \left(D_{a^{+}}^{\alpha} f\right)(x)=D\left(I_{a^{+}}^{1-\alpha} f\right)(x), x>a .
\end{aligned}
$$

Analogous formulas yield the right-sided and left-sided Liouville-Caputo derivatives of order $\alpha$, respectively:

$$
\begin{aligned}
& \left({ }^{C} D_{b^{-}}^{\alpha} f\right)(x)=\left(I_{b^{-}}^{1-\alpha}(-D) f\right)(x), x<b, \\
& \left({ }^{C} D_{a^{+}}^{\alpha} f\right)(x)=\left(I_{a^{+}}^{1-\alpha} D f\right)(x), x>a .
\end{aligned}
$$

Property 1: Let $f, g \in C[a, b]$. Then, the fractional differential operators defined in (2.3)-(2.5) satisfy the following identities:

$$
\text { (i) } \int_{a}^{b} f(x) D_{b^{-}}^{\alpha} g(x) d x=\int_{a}^{b} g(x)^{C} D_{a^{+}}^{\alpha} f(x) d x-\left.f(x) I_{b^{-}}^{1-\alpha} g(x)\right|_{a} ^{b},
$$




$$
\text { (ii) } \int_{a}^{b} f(x) D_{a^{+}}^{\alpha} g(x) d x=\int_{a}^{b} g(x)^{C} D_{b^{-}}^{\alpha} f(x) d x+\left.f(x) I_{a^{+}}^{1-\alpha} g(x)\right|_{a} ^{b} \text {. }
$$

Property 2 (see [11]): Assume that $\alpha \in(0,1), \beta>\alpha$, and $f \in C[a, b]$. Then the relations

$$
\begin{aligned}
D_{a^{+}}^{\alpha} I_{a^{+}}^{\alpha} f(x) & =f(x), \\
{ }^{C} D_{a^{+}}^{\alpha} I_{a^{+}}^{\alpha} f(x) & =f(x), \\
D_{a^{+}}^{\alpha} I_{a^{+}}^{\beta} f(x) & =I_{a^{+}}^{\beta-\alpha} f(x), \\
D_{b^{+}}^{\alpha} I_{b^{-}}^{\beta} f(x) & =I_{b^{-}}^{\beta-\alpha} f(x), \\
D_{b^{-}}^{\alpha} I_{b^{-}}^{\alpha} f(x) & =f(x), \\
{ }^{C} D_{b^{-}}^{\alpha} I_{b^{-}}^{\alpha} f(x) & =f(x),
\end{aligned}
$$

hold for any $x \in[a, b]$. Furthermore, the integral operators defined in (2.1)-(2.2) satisfy the following semi-group properties:

$$
\begin{aligned}
& I_{a^{+}}^{\alpha} I_{a^{+}}^{\beta}=I_{a^{+}}^{\alpha+\beta} ; \\
& I_{b^{-}}^{\alpha} I_{b^{-}}^{\beta}=I_{b^{-}}^{\alpha+\beta} .
\end{aligned}
$$

Now, we introduce convenient Hilbert space $L_{\omega}^{2}((a, b) ; E)\left(E:=\mathbb{C}^{2}\right)$ of vectorvalued functions using the inner product

$$
\begin{aligned}
(f, g) & :=\int_{a}^{b} f_{1}(x) \overline{g_{1}(x)} \omega_{1}(x) d x \\
& +\int_{a}^{b} f_{2}(x) \overline{g_{2}(x)} \omega_{2}(x) d x
\end{aligned}
$$

where

$$
f(x)=\left(\begin{array}{l}
f_{1}(x) \\
f_{2}(x)
\end{array}\right), g(x)=\left(\begin{array}{l}
g_{1}(x) \\
g_{2}(x)
\end{array}\right),
$$

$f_{i}, g_{i}$ and $\omega_{i}$ are real-valued continuous functions defined on $[a, b]$ and $\omega_{i}(x)>$ $0, \forall x \in[a, b],(i=1,2)$.

\section{Main Results}

In the present section, our goal is to study the fractional Dirac type system which includes the right-sided Liouville-Caputo and the left-sided Riemann-Liouvile fractional derivatives of same order $\alpha$. Throughout this section, we assume $\alpha \in(0,1)$.

Let

$$
\begin{gathered}
\Upsilon y=\left(\begin{array}{cc}
0 & { }^{C} D_{b^{-}}^{\alpha} \\
D_{a^{+}}^{\alpha} & 0
\end{array}\right)\left(\begin{array}{c}
y_{1} \\
y_{2}
\end{array}\right)+\left(\begin{array}{cc}
p(x) & 0 \\
0 & r(x)
\end{array}\right)\left(\begin{array}{l}
y_{1} \\
y_{2}
\end{array}\right) \\
=\left(\begin{array}{c}
{ }^{C} D_{b^{-}}^{\alpha} y_{2}+p(x) y_{1} \\
D_{a^{+}}^{\alpha} y_{1}+r(x) y_{2}
\end{array}\right),
\end{gathered}
$$


where $y:=\left(\begin{array}{l}y_{1} \\ y_{2}\end{array}\right)$. With this notation, we consider the fractional Dirac type system:

$$
\Upsilon y_{\lambda}=\lambda \omega y_{\lambda}, a \leq x \leq b<\infty
$$

where $y_{\lambda}=\left(\begin{array}{l}y_{\lambda 1} \\ y_{\lambda 2}\end{array}\right), p, r$ are real-valued continuous functions defined on $[a, b], \omega(x)=$ $\left(\begin{array}{cc}\omega_{1}(x) & 0 \\ 0 & \omega_{2}(x)\end{array}\right), \omega_{i}$ are real-valued continuous functions defined on $[a, b]$ and $\omega_{i}(x)>$ $0, \forall x \in[a, b],(i=1,2), \lambda$ is a complex spectral parameter and boundary conditions

$$
\begin{aligned}
& a_{11} I_{a^{+}}^{1-\alpha} y_{\lambda 1}(a)+a_{12} y_{\lambda 2}(a)=0 \\
& a_{21} I_{a^{+}}^{1-\alpha} y_{\lambda 1}(b)+a_{22} y_{\lambda 2}(b)=0
\end{aligned}
$$

with $a_{11}^{2}+a_{12}^{2} \neq 0$ and $a_{21}^{2}+a_{22}^{2} \neq 0$.

Theorem 3.1. The operator $T:=\omega^{-1} \Upsilon$ generated by fractional Dirac type system (FD) defined by (3.1)-(3.3) is formally self-adjoint on $L_{\omega}^{2}((a, b) ; E)$.

Proof. Let $y(),. z(.) \in L^{2}((a, b) ; E)$. Then, we have

$$
\begin{gathered}
(T y, z)-(y, T z)=\int_{a}^{b}\left(D_{a^{+}}^{\alpha} y_{1}+r(x) y_{2}\right) \overline{z_{2}} d x \\
+\int_{a}^{b}\left({ }^{C} D_{b^{-}}^{\alpha} y_{2}+p(x) y_{1}\right) \overline{z_{1}} d x \\
-\int_{a}^{b} y_{2} \overline{\left(D_{a^{+}}^{\alpha} z_{1}+r(x) z_{2}\right)} d x \\
-\int_{a}^{b} y_{1} \overline{\left({ }^{C} D_{b^{-}}^{\alpha} z_{2}+p(x) z_{1}\right)} d x \\
=\int_{a}^{b}\left(D_{a^{+}}^{\alpha} y_{1}\right) \overline{z_{2}} d x+\int_{a}^{b}\left({ }^{C} D_{b^{-}}^{\alpha} y_{2}\right) \overline{z_{1}} d x \\
-\int_{a}^{b} y_{2} \overline{\left(D_{a^{+}}^{\alpha} z_{1}\right)} d x-\int_{a}^{b} y_{1} \overline{\left({ }^{C} D_{b^{-}}^{\alpha} z_{2}\right)} d x
\end{gathered}
$$

Since

$$
\begin{gathered}
\int_{0}^{a}\left({ }^{C} D_{b^{-}}^{\alpha} y_{2}\right) \overline{z_{1}} \omega_{1} d x=\int_{a}^{b} y_{2} \overline{\left(D_{a^{+}}^{\alpha} z_{1}\right)} \omega_{1} d x \\
-\left[y_{2}(b) \overline{I_{a^{+}}^{1-\alpha} z_{1}(b)}-y_{2}(a) \overline{I_{a^{+}}^{1-\alpha} z_{1}(a)}\right]
\end{gathered}
$$

and

$$
\begin{gathered}
\int_{a}^{b} y_{1} \overline{\left({ }^{C} D_{b^{-}}^{\alpha} z_{2}\right)} d x=\int_{a}^{b}\left(D_{a^{+}}^{\alpha} y_{1}\right) \overline{z_{2}} d x \\
-\left[\overline{z_{2}(b)} I_{a^{+}}^{1-\alpha} y_{1}(b)-\overline{z_{2}(a)} z_{2}(a) I_{a^{+}}^{1-\alpha} y_{1}(a)\right]
\end{gathered}
$$


we get

$$
(T y, z)-(y, T z)=[y, z]_{b}-[y, z]_{a},
$$

where $[y, z]_{x}:=\overline{z_{2}(x)} I_{a^{+}}^{1-\alpha} y_{1}(x)-y_{2}(x) \overline{I_{a^{+}}^{1-\alpha} z_{1}(x)}$. We proceed to show that the equality $(T y, z)=(y, T z)$ for any $y(),. z(.) \in L^{2}((a, b) ; E)$. From the boundary conditions (3.2) and (3.3), we get $[y, z]_{b}=0$ and $[y, z]_{a}=0$. Consequently,

$$
(T y, z)=(y, T z) \text {. }
$$

This completes the proof.

Lemma 3.1. All eigenvalues of the FD system defined by (3.1)-(3.3) are real.

Proof. Let $\mu$ be an eigenvalue with an eigenfunction $z(x)$. From the equality (3.5), we get

$$
(T z, z)=(z, T z)=(z, \mu z)=\bar{\mu}(z, z)
$$

On the other hand,

$$
(T z, z)=(\mu z, z)=\mu(z, z) .
$$

It follows from (3.6) and (3.7) that

$$
\mu(z, z)=\bar{\mu}(z, z), \quad(\mu-\bar{\mu})(z, z)=0 .
$$

Since $z(x) \neq 0$, we get $\mu=\bar{\mu}$.

Lemma 3.2. If $\mu_{1}$ and $\mu_{2}$ are two different eigenvalues of the FD system defined by (3.1)-(3.3), then the corresponding eigenfunctions $\theta$ and $\eta$ are orthogonal in the space $L_{\omega}^{2}((a, b) ; E)$.

Proof. Let $\mu_{1}$ and $\mu_{2}$ be two different real eigenvalues with corresponding eigenfunctions $\theta$ and $\eta$, respectively. From (3.5), we obtain

$$
(T \theta, \eta)=(\theta, T \eta),\left(\mu_{1} \theta, \eta\right)=\left(\theta, \mu_{2} \eta\right),\left(\mu_{1}-\mu_{2}\right)(\theta, \eta)=0 .
$$

Since $\mu_{1} \neq \mu_{2}$, we obtain that $\theta(x)$ and $\eta(x)$ are orthogonal in $L_{\omega}^{2}((a, b) ; E)$.

$$
\text { Now let } y(x)=\left(\begin{array}{c}
y_{1}(x) \\
y_{2}(x)
\end{array}\right), z(x)=\left(\begin{array}{c}
z_{1}(x) \\
z_{2}(x)
\end{array}\right) \in L^{2}((a, b) ; E) \text {. Then, we }
$$
define the Wronskian of $y(x)$ and $z(x)$ by

$$
W(y, z)(x)=I_{a^{+}}^{1-\alpha} y_{1}(x) z_{2}(x)-I_{a^{+}}^{1-\alpha} z_{1}(x) y_{2}(x) .
$$


Theorem 3.2. The Wronskian of any solution of Eq. (3.1) is independent of $x$.

Proof. Let $y(x)$ and $z(x)$ be two solutions of Eq. (3.1). By Green's formula (3.4), we have

$$
(T y, z)-(y, T z)=[y, z]_{b}-[y, z]_{a} .
$$

Since $T y=\lambda y$ and $T z=\lambda z$, we have

$$
\begin{aligned}
(\lambda y, z)-(y, \lambda z) & =[y, z]_{b}-[y, z]_{a} \\
(\lambda-\bar{\lambda})(y, z) & =[y, z]_{b}-[y, z]_{a} .
\end{aligned}
$$

Since $\lambda \in \mathbb{R}$, we have $[y, z]_{b}=[y, z]_{a}=W(y, \bar{z})(a)$, i.e., the Wronskian is independent of $x$.

Corollary 3.1. If $y(x)$ and $z(x)$ are both solutions of Equation (3.1), then either $W(y, z)(x)=0$ or $W(y, z)(x) \neq 0$ for all $x \in[a, b]$.

Theorem 3.3. Any two solutions of the equation (3.1) are linearly dependent if and only if their Wronskian is zero.

Proof. Let $y(x)$ and $z(x)$ be two linearly dependent solutions of Equation (3.1). Then, there exists a constant $c>0$ such that $y(x)=c z(x)$. Hence

$$
W(y, z)=\left|\begin{array}{cc}
I_{a^{+}}^{1-\alpha} y_{1}(x) & y_{2}(x) \\
I_{a^{+}}^{1-\alpha} z_{1}(x) & z_{2}(x)
\end{array}\right|=\left|\begin{array}{cc}
c I_{a^{+}}^{1-\alpha} z_{1}(x) & c z_{2}(x) \\
I_{a^{+}}^{1-\alpha} z_{1}(x) & z_{2}(x)
\end{array}\right|=0 .
$$

Conversely, the Wronskian $W(y, z)=0$ and therefore, $y(x)=c z(x)$, i.e., $y(x)$ and $z(x)$ are linearly dependent.

Before proceeding further, we need the following auxiliary functions.

We introduce the function $\phi(x):=\left(\begin{array}{c}\left(I_{a+1}^{\alpha} 1\right)(x) \\ \left(I_{b^{-}}^{\alpha} 1\right)(x)\end{array}\right)$. Further, the general solution of the equation $\Upsilon \psi=0$, i.e.,

$$
\left(\begin{array}{cc}
0 & { }^{C} D_{b^{-}}^{\alpha} \\
D_{a^{+}}^{\alpha} & 0
\end{array}\right)\left(\begin{array}{l}
\psi_{1} \\
\psi_{2}
\end{array}\right)=0
$$

is given by

$$
\psi=\left(\begin{array}{c}
\xi_{1} \Phi(\alpha, a, x) \\
\xi_{2}
\end{array}\right)
$$

where

$$
\Phi(\alpha, a, x)=\frac{(x-a)^{\alpha-1}}{\Gamma(\alpha)}
$$


Lemma 3.3. Let

$$
\Delta:=a_{11} a_{12}-a_{11} a_{21}
$$

and

$$
Y_{\lambda}(y):=\{V-\lambda \omega\} y_{\lambda}
$$

where $V(x):=\left(\begin{array}{cc}p(x) & 0 \\ 0 & r(x)\end{array}\right)$. Assume $\Delta \neq 0$. Then on the space $C[a, b]$, the FD system defined by (3.1)-(3.3) is equivalent to the integral equation

$$
y_{\lambda}(x)=-M Y_{\lambda}(y)+A(x) T+B(x) Z,
$$

where the coefficients $M, A, T, B$ and $Z$ are

$$
\begin{aligned}
& M:=\left(\begin{array}{cc}
0 & I_{a^{+}}^{\alpha} \\
I_{b^{-}}^{\alpha} & 0
\end{array}\right), \\
& A(x) \quad:=\left(\begin{array}{c}
\frac{a_{12} a_{22}}{\Delta} \Phi(\alpha, a, x) \\
-\frac{a_{21} a_{12}}{\Delta}
\end{array}\right), \\
& T \quad:=-\left.I_{b^{-}}^{\alpha} Y_{\lambda 1}(y)\right|_{x=a}, \\
& B(x) \quad:=\left(\begin{array}{l}
-\frac{a_{12} a_{21}}{\Delta} \Phi(\alpha, a, x) \\
\frac{a_{21} a_{11}}{\Delta}
\end{array}\right), \\
& Z \quad:=-\left.I_{a^{+}}^{1} Y_{\lambda 2}(y)\right|_{x=b},
\end{aligned}
$$

and the function $\Phi(\alpha, a, x)$ is defined in (3.8).

Proof. Using fractional composition rules and (3.9), we can rewrite the equation (3.1) as follows:

$$
\Upsilon\left[y_{\lambda}(x)+M Y_{\lambda}(y)\right]=0 .
$$

Thus, we get

$$
y_{\lambda}(x)+M Y_{\lambda}(y)=\left(\begin{array}{c}
\xi_{1} \Phi(\alpha, a, x) \\
\xi_{2}
\end{array}\right)
$$

i.e.,

$$
y_{\lambda}(x)=-M Y_{\lambda}(y)+\left(\begin{array}{c}
\xi_{1} \Phi(\alpha, a, x) \\
\xi_{2}
\end{array}\right)
$$

Now, we shall connect the coefficients $\xi_{i}(i=1,2)$ to the values $a_{i j}(i, j=1,2)$ in the boundary conditions (3.2)-(3.3). From the equation (3.10), we obtain

$$
K y_{\lambda}(x)=-K M Y_{\lambda}(y)+K\left(\begin{array}{c}
\xi_{1} \Phi(\alpha, a, x) \\
\xi_{2}
\end{array}\right)
$$


where $K:=\left(\begin{array}{cc}I_{a^{+}}^{1-\alpha} & 0 \\ 0 & 1\end{array}\right)$. Then we have

$$
\left(\begin{array}{c}
I_{a^{+}}^{1-\alpha} y_{\lambda 1} \\
y_{\lambda 2}
\end{array}\right)=-\left(\begin{array}{cc}
0 & I_{a^{+}}^{1} \\
I_{b^{-}}^{\alpha} & 1
\end{array}\right) Y_{\lambda}(y)+\left(\begin{array}{c}
I_{a^{+}}^{1-\alpha}\left[\xi_{1} \Phi(\alpha, a, x)\right] \\
\xi_{2}
\end{array}\right)
$$

i.e.,

$$
\left(\begin{array}{c}
I_{a^{+}}^{1-\alpha} y_{\lambda 1} \\
y_{\lambda 2}
\end{array}\right)=\left(\begin{array}{c}
-I_{a^{+}}^{1} Y_{\lambda 2}(y) \\
-I_{b^{-}}^{\alpha} Y_{\lambda 1}(y)
\end{array}\right)+\left(\begin{array}{l}
\xi_{1} \\
\xi_{2}
\end{array}\right)
$$

By virtue of (3.2) and (3.3), we conclude that

$$
\begin{array}{rc}
I_{a^{+}}^{1-\alpha} y_{\lambda 1}(a) & =\xi_{1}, \\
I_{a^{+}}^{1-\alpha} y_{\lambda 1}(b) & =-\left.I_{a^{+}}^{1} Y_{\lambda 2}(y)\right|_{x=b}+\xi_{1}, \\
y_{\lambda 2}(a) & =-\left.I_{b^{-}}^{\alpha} Y_{\lambda 1}(y)\right|_{x=a}+\xi_{2}, \\
y_{\lambda 2}(b) & =\xi_{2} .
\end{array}
$$

This leads to the system of equations

$$
\begin{aligned}
& a_{11} \xi_{1}+a_{12} \xi_{2}=a_{12} T \\
& a_{21} \xi_{1}+a_{22} \xi_{2}=a_{12} Z .
\end{aligned}
$$

Since $\Delta \neq 0$, the solution for coefficients $\xi_{j}, j=1,2$ is unique:

$$
\begin{aligned}
& \xi_{1}=\frac{a_{11}\left(a_{22} T-a_{21} Z\right)}{\Delta}, \\
& \xi_{2}=\frac{a_{21}\left(a_{11} Z-a_{12} T\right)}{\Delta} .
\end{aligned}
$$

We have finished the proof of the lemma.

Now, we prove the existence and uniqueness of eigenfunction of the regular FD system defined by (3.1)-(3.3). In the next result, we use the following notation:

$$
A:=\|A(x)\|_{C}, B:=\|B(x)\|_{C}, S_{\phi}:=\|\phi(x)\|_{C},
$$

where $\|\cdot\|_{C}$ denotes the supremum norm on the space $C([a, b], E)$.

Theorem 3.4. Let $\alpha \in(0,1)$ and assume $\Delta \neq 0$. Then unique continuous function $y_{\lambda}$ for the regular FD system defined by (3.1)-(3.3) corresponding to each eigenvalue obeying

$$
\|V-\lambda \omega\|_{C} \leq \frac{1}{S_{\phi}+A\|\phi(a)\|_{C}+B(b-a)}
$$

exists and such eigenvalue is simple. 
Proof. Let us define the mapping $L: C([a, b], E) \rightarrow C([a, b], E)$ by

$$
L f:=-M Y_{\lambda}(f)+A(x) T+B(x) Z,
$$

Now, we show that the equation (3.1) can be interpreted as a fixed point condition on the space $C([a, b], E)$. Using the following estimate

$$
\left\|Y_{\lambda}(g)-Y_{\lambda}(h)\right\|_{C} \leq\|g-h\|_{C}\|V-\lambda \omega\|_{C},
$$

we conclude that

$$
\begin{gathered}
\|L g-L h\|_{C} \quad \leq\|g-h\|_{C}\|V-\lambda \omega\|_{C} S_{\phi}+A\|g-h\|_{C}\|\phi(a)\|_{C} \\
+B(b-a)\|g-h\|_{C}\|V-\lambda \omega\|_{C} \\
=\|V-\lambda \omega\|_{C}\|g-h\|_{C}\left(S_{\phi}+A\|\phi(a)\|_{C}+B(b-a)\right) \\
=\Pi\|g-h\|_{C}
\end{gathered}
$$

where $\Pi=\|V-\lambda \omega\|_{C}\left(S_{\phi}+A\|\phi(a)\|_{C}+B(b-a)\right)$. By the condition (3.11), the mapping $L$ is a contraction on the space $C([a, b], E)$ so it has a unique fixed point. Therefore, such eigenvalue is simple.

\section{REFEREN C ES}

1. B. P. Allahverdiev and H. Tuna: q-fractional Dirac type systems. Rad Hrvat. Akad. Znan. Umjet. Mat. Znan. Vol. 24 / 542 (2020): 117-130.

2. E. BAŞ: Fundamental spectral theory of fractional singular Sturm-Liouville operator, Journal of Function Spaces and Applications, Volume 2013, Article ID 915830, (2013) 7 pages.

3. E. BAŞ and F. Metin: Fractional singular Sturm-Liouville operator for Coulomb potential, Advances in Difference Equations, 2013:300, (2013).

4. F.Z. Bensidhoum and H. DiB: On some regular fractional Sturm-Liouville problems with generalized Dirichlet conditions, J. Integral Equations Applications 28, no. 4, (2016) 459-480.

5. J. C. Cuenin: Eigenvalue bounds for Dirac and fractional Schrödinger operators with complex potentials, Journal of FunctionalAnalysis, 272 (2017), 2987-3018.

6. M. H. Derakhshan and A. Ansari: Fractional Sturm-Liouville problems for Weber fractional derivatives, International Journal of Computer Mathematics, 96:2, (2019) 217-237.

7. M. FERREIRA and N. VIEIRA: Eigenfunctions and fundamental solutions of the fractional Laplace and Dirac operators: the Riemman-Liouville case, Complex Anal. Oper. Theory, 10 (5), (2016), 1081-1100.

8. M. Ferreira and N. Vieira: Eigenfunctions and Fundamental Solutions of the Caputo Fractional Laplace and Dirac Operators, Modern Trends in Hypercomplex Analysis, Trends in Mathematics, (2016),191-202.

9. R. Hilfer: Ed., Applications of Fractional Calculus in Physics, World Scientific, Singapore, 2000. 
10. A. A. Kilbas, H. M. Srivastava and J. J. Trujillo: Theory and Applications of Fractional Differential Equations, vol. 204, Elsevier, Amsterdam, The Netherlands, 2006

11. M. Klimek and O. P. ArgawAl: Regular fractional Sturm-Liouville problem with generalized derivatives of order in $(0,1)$, In: Proceedings of the IFAC Joint Conference: 5th SSSC, 11th WTDA, 5th WFDA, 4-6 February 2013, Grenoble, France.

12. M. KlimeK and O. P. ARGawAL: Fractional Sturm-Liouville problem, Computers and Mathematics with Applications, 66, (2013) 795-812.

13. M. Klimek, T. OdziJewicz and A. B. Malinowska: Variational methods for the fractional Sturm-Liouville problem, J. Math. Anal. Appl. 416 , (2014), 402-426.

14. M. Klimke and M. BlasiK: Regular fractional Sturm-Liouville problem with discrete spectrum: solutions and applications, In: Proceedings of the 2014 International Conference on Fractional Differentiaton and Its Applications, 23-25 June 2014, Catania, Italy

15. M. Klimek, A. B. Malinowska and T. OdziJewicz: Applications of the fractional Sturm-Liouville problem to the space-time fractional diffusion in finite domain, Fract. Calc. Appl. Anal. 19(2) (2016), 516-550.

16. V. LAKSHMikANThAM and A. S. VATSALA: Basic theory of fractional differential equations, Nonlinear Anal. TMA 698 (2008) 2677-2683.

17. V. LAKShmikAntham and A. S. VATSAla: Theory of fractional differential inequalities and applications, Commun. Appl. Anal. 11 (2007) 395-402.

18. B. M. Levitan and I. S. SARgsjan: Introduction to Spectral Theory: Self adjoint Ordinary Differential Operators, American Mathematical Society, Providence, RI, USA, 1975 .

19. K. S. Miller and B. Ross: An Introduction to the Fractional Calculus and Fractional Differential Equations, John Wiley \& Sons, New York, NY, USA, 1993.

20. I. Poblubny: Fractional Differential Equations, vol. 198, Academic Press, San Diego, CA, USA, 1999.

21. M. Rivero, J.J. Trujillo and M. P. Velasco: A fractional approach to the SturmLiouville problem, Cent.Eur.J.Phys. 11(10), (2013), 1246-1254.

22. S. G. Samko, A. A. Kilbas and O. I. Marichev: Fractional Integral and Derivatives, Theory and Applications, Gordon and Breach, Switzerland, 1993.

23. H. M. SRIVASTAVA: Fractional-Order Derivatives and Integrals: Introductory Overview and Recent Developments, Kyungpook Mathematical Journal, 60 (1), (2020), 73-116.

24. H. M. SRIVAStava: Diabetes and its resulting complications: Mathematical modeling via fractional calculus, Public Health Open Access, 4(3), (2020), Article ID 2, 1-5.

25. B. Thaller: The Dirac Equation, Springer-Verlag, Berlin Heidelberg, 1992.

26. J. Weidmann: Spectral Theory of Ordinary Differential Operators. Lecture Notes in Mathematics 1258, Springer, Berlin, 1987.

27. M. ZAYERNOURI and G.E. KARNIADAKIS: Fractional Sturm-Liouville eigen-problems: Theory and numerical approximation, J. Comput. Phys. 252(1), (2013),495-517. 